Relations industrielles

Industrial Relations

\title{
An American Philosophy of Social Security, by J. Douglas Brown, Princeton, Princeton University Press, Toronto, Saunders of Toronto Ltée, 1972, 244 pp.
}

\section{Gilles Beausoleil}

Volume 28, numéro 4, 1973

URI : https://id.erudit.org/iderudit/028465ar

DOI : https://doi.org/10.7202/028465ar

Aller au sommaire du numéro

Éditeur(s)

Département des relations industrielles de l'Université Laval

ISSN

0034-379X (imprimé)

1703-8138 (numérique)

Découvrir la revue

Citer ce compte rendu

Beausoleil, G. (1973). Compte rendu de [An American Philosophy of Social Security, by J. Douglas Brown, Princeton, Princeton University Press, Toronto, Saunders of Toronto Ltée, 1972, 244 pp.] Relations industrielles / Industrial Relations, 28(4), 894-896. https://doi.org/10.7202/028465ar

Tous droits réservés @ C Département des relations industrielles de l'Université Laval, 1973
Ce document est protégé par la loi sur le droit d'auteur. L’utilisation des services d'Érudit (y compris la reproduction) est assujettie à sa politique d'utilisation que vous pouvez consulter en ligne.

https://apropos.erudit.org/fr/usagers/politique-dutilisation/ 
what type of model would generate a wage-push inflation. In part Rees attributes it to a growth in union power (particularly to militant public employees) but his argument appears to be intended for application in a situation where union power is constant. The former is understandable but the latter seems to require a revised labor market model particularly since Friedman and others have argued that a constant level of monopoly power has no implications at all for price or wage changes. However, the chapter is, in general, an interesting approach to what Rees obviously recognizes as an important unresolved problem.

This is an outstanding book which can be used profitably by those who wish to acquaint themselves with recent developments in labor economics and by students at a variety of levels who will be grateful for a highly lucid presentation of some relatively difficult points.

\section{Carleton University}

Douglas A. SMVTH

An American Philosophy of Social Security, by J. Douglas Brown, Princeton, Princeton University Press, Toronto, Saunders of Toronto Ltee, 1972, $244 \mathrm{pp}$

L'auteur de ce volume a été intimement associé au développement de la sécurité sociale américaine. Il y a consacré nombre de ses travaux et il a participé à de multiples groupes de travail impliqués dans l'élaboration des politiques de sécurité sociale. A partir de son expérience, le professeur Brown s'est donné comme objectif de rédiger un volume de synthèse sur le développement de la sécurité sociale américaine. Ce volume s'adresse à des lecteurs de formation et d'expérience générales plutôt qu'à des experts dans ce domaine. L'auteur vise à éclairer l'opinion publique sur les dimensions importantes de la politique américaine de sécurité sociale.

Dans l'ensemble, l'auteur a atteint l'objectif qu'il recherchait. Le volume couvre differents aspects de la sécurité sociale américaine. Ces aspects sont les suivants : L'évolution de la politique américaine de sécurité sociale (Chapitre 1, 2, 3); la place du régime principal de la sécurité sociale dans l'ensemble du système (Chapitres 4, 5); les éventualités couvertes et les personnes protégées (Chapitres 8 , $9,10,11,12)$; le financement du régime (Chapitres $6,7,13$ ); et la sécurité sociale de la santé (Chapitres 14,15).

Les considérations judicieuses reposant sur une bonne information de l'auteur apportent à cette description du régime américain un contenu de bonne qualité. Le lecteur peut ainsi comprendre dans quel contexte économique et social les conséquences de la dépression américaine des années '30 ont amené le gouvernement américain à mettre en place un régime de sécurité sociale dont les deux pièces maîtresses furent celles du régime de retraite pour les personnes âgées et les invalides et l'assurance-chômage. $\mathrm{Vu}$ que seulement le premier régime s'est établi à l'échelle nationale et que l'auteur y a été associé personnellement, il limite la description de la sécurité sociale à ce seul programme majeur.

Par la suite, l'auteur établit la relation entre ce programme et les autres modes généraux de couverture du risque de la dépendance sociale soit ceux de l'assistance sociale pour les indigents et de l'assurance privée pour les personnes touchant des revenus supérieurs à la moyenne L'auteur précise le rôle des assurances sociales, les caractéristiques d'une politique établissant correctement les points-frontières entre l'assurance sociale publique et l'assistance sociale ainsi que les assurances privées. Il décrit ensuite les catégories de personnes protégées par le régime américain, soit les personnes âgées, les dépendants de ces dernières et les invalides. Il revoit les raisons qui ont amené le gouvernement américain à établir l'âge de l'accessibilité au programme, la structure des allocations, les barèmes relatifs à ces allocations et les problèmes généraux de financement dont ceux des contributions des employeurs et des employés, du niveau de la réserve financière et de la participation possible du gouvernmeent et de l'utilisation des fonds du régime.

Les deux chapitres consacrés à la santé éclairent le lecteur sur ce que l'auteur appelle la longue et dure lutte de la population américaine pour se donner un programme d'assurance maladie couvrant certains risques associés aux mauvais états de santé. L'auteur signale dès le 
début de la section consacrée à ce sujet que cette lutte n'a eu qu'un succès mitigé et que le programme américain d'assurance maladie ne couvre qu'une partie de la population et qu'il se limite au financement des soins hospitaliers sur une base obligatoire et des soins médicaux sur une base volontaire. Le programme est donc fragmenté et il ne comporte pas les mécanismes appropriés pour établir un régime général de soins basés sur une allocation efficace des ressources et comportant des soins de qualité. De fait, ce régime imparfait ne date d'ailleurs que de l'année 1964, c'est-à-dire qu'il a été établi plus d'un quart de siècle après l'établissement de la caisse de retraite américaine.

Dans sa conclusion, l'auteur signale que la construction de l'édifice de la sécurité sociale américaine est avancée dans le sens que des programmes existent mais que ces programmes sont incomplets et ne sont pas intégrés dans un ensemble harmonieux et susceptible de répondre aux besoins de la collectivité américaine. L'auteur signale que cette construction sera achevée par l'instauration d'un régime d'assurance maladie complet et par l'intégration dans une politique générale de sécurité du revenu d'un régime national d'assistance publique et d'assurancechômage à l'actuel régime de retraite et de rentes qui existe aux Etats-Unis.

$\mathrm{Au}$ plan méthododolique, la méthode descriptive utilisée par l'auteur dans la majeure partie de son étude lui sert bien, particulièrement pour décrire le conter: du programme américain et son évolution historique. Le peu de place faite à l'utilisation d'une approche analytique ou de modèles spécifiques soulève certains problèmes sérieux au plan des positions de l'auteur ou de l'étude même des phénomènes. Cette faiblesse analytique donne lieu à des jugements de valeur sur leri1cacité de la structure américaine de sécurité sociale. L'auteur semble aussi accorder une valeur absolue aux programmes nationaux de sécurité sociale sans faire mention des difficultés relatives à de tels programmes dans une société où les disparités sont considérables et les possibilités de participation de la population à des programmes nationaux fort limitées. L'absence de comparaisons internationales est assez uepurable dans une étude de ce genre. De plus, il est surprenant que le recours à l'analyse économique soit aussi faible dans le cas d'un sujet qui touche aussi directement à la distribution du revenu que celui traité par l'auteur.

Le principal commentaire qui peut cependant être fait et qui constitue un jugement sur cette œuvre me semble celui d'un certain anachronisme. Sauf pour l'inclusion de données récentes ou d'observations sur l'évolution historique, ce volume aurait pu être écrit il y a 20 ans tout aussi bien qu'en 1970. En effet, dans ses sources théoriques ou analytiques, l'œuvre ne fait pas appel à l'appareil conceptuel des travaux modernes dans ce domaine. Il n'y est nullement mention du courant de recherche américaine des années récentes sur la distribution, la redistribution du revenu et la pauvreté. De plus, dans son évaluation du programme américain, l'auteur ne fait pas référence aux multiples travaux rattachés directement ou indirectement à la rationalisation des choix budgétaires.

Cette dernière approche permet mainienant des évaluations systématiques des décisions collectives ou privées grâce à un appareil scientifique de plus en plus perfectionné. De même, il est suprenant de ne pas trouver dans cet ouvrage des références aux divers travaux récents sur la structure de la sécurité sociale, sur la place d'un programme de revenu garanti dans la sécurité sociale contemporaine sur lequel tant de travaux américains ont porté au cours des années récentes. L'absence de mention des caractéristiques fondamentales de la sécurité sociale dans les pays occidentaux contemporains présente aussi des éléments de surprise pour un lecteur averti.

En terminant, quelques considérations constituant pratiquement des postulats pour le professeur Brown doivent faire l'objet d'une mention critique. En premier lieu, le titre même du volume est erroné puisqu'il ne traite que d'un ensemble de programmes de redistribution de revenu pour les personnes hors du marché du travail. Cet ensemble ne correspond pas à la structure intégrale de la sécurité sociale reconnue dans tous les pays contemporains. Les allocations familiales, l'assurance-chômage, les accidents du travail, la continuité du revenu dans les cas de maladie ne sont peu ou pas mentionnés dans l'étude du professeur Brown. Un deuxième aspect se rap- 
porte à la philosophie progressive dont l'auteur fait état en traitant du programme américain de retraite et de rentes. Il aurait été utile de réconcilier ce caractère progressif avec les travaux sur la pauvreté qui encore tout récemment font état d'une incidence élevée de la pauvreté dans le cas des catégories de population couvertes par ce programme, particulièrement dans le cas des personnes âgées. Un aspects additionnel se rapporte à la foi qu'exprime l'auteur dans l'efficacité des programmes nationaux sans tenir compte des inéquités que peuvent présenter les normes nationales pour des populations faisant face à des différences considérables du coût de vie ou de revenus moyens L'auteur aurait aussi pu éviter de développer la notion de la neutralité de l'administration de la sécurité sociale dans l'utilisation des fords de réserve surtout qu'il signale que le placement des fonds comporte exclusivement l'achat d'obligations du gouvernement fédéral. Une lecture naïve de cette affirmation est que le programme a certes favorisé le gouvernement fédéral par rapport aux gouvernements des états et des municipalités dont les responsabilités en matière de programmes sociaux sont considérables aux Etats-Unis.

Dans l'ensemble, ce volume est utile et intéressant. Bien qu'apologétique, il ne nous éclaire pas sur les conditions sociales et économiques contemporaines dans lesquelles s'insère la sécurité sociale américaine, sur les problèmes fondamentaux de redistribution de revenu outre-frontière et sur les options prévisibles de cette sécurité sociale qui seront certes prises par le milieu amérisain dans le futur.

Conseil économique du Canada

Gilles BEAUSOLEIL

Les nouveaux marchands d'lommes? Etude du travail intérimaire, par Guy Caire, Paris, Editions ouvrières-Economie et Humanisme, 1973, 255 pp.

La notoriété de l'auteur, professeur de sciences économiques à l'Université de Paris X-Nanterre, n'est plus à faire. Il est l'auteur d'une cuvre abondante et de grande qualité, dont plusieurs ouvrages concernent la planification, la politique des revenus et les syndicats ouvriers, ce dernier, en somme, étant inspiré de «l'approche dite des relations industrielles», dont «l'esprit est résolument interdisciplinaire ».

C'est également dans la "perspective des relations industrielles »- dont l'auteur définit l'optique (pp. 12-15) - que s'inscrit cette étude du travail intérimaire, qui devient désormais le livre de réféfence en la matière. Après avoir explicité dans l'introduction la notion de travail intérimaire par rapport à celle, substituable, de travail temporaire et à celle, non substituable, de travail à temps partiel, M. Caire expose dans une première partie les particularités du marché du travail intérimaire sous un triple biais: statistique (essor dans l'espace et croissance dans le temps), socio-économique (point de vue des firmes utilisatrices et celui du personnel intermédiaire) et juridique (la nature de la relation triangulaire et le statut du travail intérimaire). La seconde partie est consacrée à l'organisation du marché du travail intérimaire et traite des inquiétudes collectives suscitées (optiques des organisations ouvrières et de la puissance publique).

L'approche interdisciplinaire choisie par l'auteur - qui fait une démonstration éloquente de ses compétences dans les diverses matières qui font des relations industrielles un domaine-carrefour - en fait un livre complet. Aucun aspect n'est éludé ni aucune optique particulière. La densité, la clarté et l'objectivité n'en sont pas le moindre mérite. Une «petite phrase» «...l'idéologie est au cơur même de la problématique des relations industrielles », annonce peut-être le prochain livre de M. Caire.

Dimitri WEISS

Université de Paris I Panthéon-Sorbonne

College Graduates and Jobs, Adjusting to a New Labor Market Situation, by The Carnegie Commission on Higher Education, New York, McGraw-Hill, 1973, 242 pp.

Les années soixante-dix seront des années plus difficiles pour les diplômés universitaires et la Commission Carnegie n'hésite pas à qualifier le tout de «a new period ». Comme elle le note cependant, c'est plutôt un retour à la normale; en effet, entre 1900 et 1950 , les écarts de traitements en faveur des personnes plus 